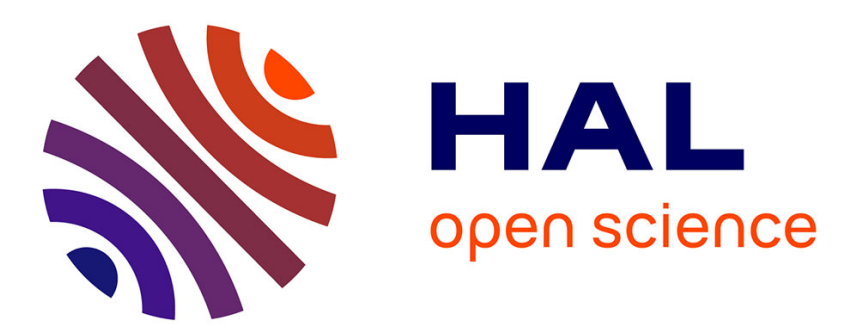

\title{
Absorption du rayonnement 12 et 8 millimètres par les vapeurs de propane sous pression
}

\author{
A. Battaglia, G. Villa, G. Boudouris
}

\section{To cite this version:}

A. Battaglia, G. Villa, G. Boudouris. Absorption du rayonnement 12 et 8 millimètres par les vapeurs de propane sous pression. Revue de Physique Appliquée, 1979, 14 (9), pp.801-805. 10.1051/rphysap:01979001409080100 . jpa-00244664

\section{HAL Id: jpa-00244664 https://hal.science/jpa-00244664}

Submitted on 1 Jan 1979

HAL is a multi-disciplinary open access archive for the deposit and dissemination of scientific research documents, whether they are published or not. The documents may come from teaching and research institutions in France or abroad, or from public or private research centers.
L'archive ouverte pluridisciplinaire HAL, est destinée au dépôt et à la diffusion de documents scientifiques de niveau recherche, publiés ou non, émanant des établissements d'enseignement et de recherche français ou étrangers, des laboratoires publics ou privés. 


\title{
REVUE DE PHYSIQUE APPLIQUÉE
}

Classification

Physics Abstracts

33.20B

\section{Absorption du rayonnement 12 et 8 millimètres par les vapeurs de propane sous pression}

\author{
A. Battaglia, G. Villa \\ Istituto di Fisica dell' Universita di Pisa, Italia \\ et G. Boudouris \\ Université Technique Nationale d'Athènes, Grèce \\ (Reçu le 12 avril 1979, accepté le 11 juin 1979)
}

\begin{abstract}
Résumé. - Etude expérimentale des pertes diélectriques $\left(\varepsilon^{\prime \prime}\right)$ présentées par le propane gazeux aux fréquences de 24 et $36 \mathrm{GHz}$, à la température ambiante et à des pressions allant jusqu'à 7 atm (liquéfaction à 9 atm environ). La discussion indique qu'une partie de l'absorption serait du type non résonnant de Debye, le reste (probablement la plupart) étant du type résonnant. Comparaison est faite avec un travail antérieur (1956) de Maryott et Birnbaum.

Abstract. - Experimental study of dielectric losses $\left(\varepsilon^{\prime \prime}\right)$ presented by gaseous propane at frequencies of 24 and $36 \mathrm{GHz}$, room temperature and pression up to $7 \mathrm{~atm}$ (liquefaction at about $9 \mathrm{~atm}$ ). The discussion shows that part of the absorption should be of the non-resonnant Debye type, the rest (probably the greatest part) being of the resonnant type. Comparison is made with a previous work of Maryott and Birnbaum (1956).
\end{abstract}

1. Introduction. - Les propriétés diélectriques des hydrocarbures saturés gazeux en très hautes fréquences ont attiré l'attention de quelques chercheurs depuis 1950 environ. Il a été constaté que, pour certaines de ces molécules (propane, isobutane, isopentane), leur symétrie structurale n'est pas incompatible avec l'existence d'un faible moment dipolaire permanent. Relever un tel moment dipolaire revient à une question de sensibilité et de précision pour le dispositif expérimental.

Le propane, en particulier, a été reconnu comme gaz absorbant en microondes par Maryott et Birnbaum (1956) [1]. Cependant, ce travail concernait surtout l'isobutane, les résultats pour le propane étant relativement sommaires et plutôt indicatifs. Quelques années après, Lide (1960) [2] et Muenter et Laurie (1966) [3] ont déterminé les constantes rotationnelles, le moment dipolaire et la structure du propane par des études spectroscopiques hertziennes en basse pression (effet Stark).

Le présent travail est une étude expérimentale de l'absorption par le propane en fonction de la pression (jusqu'à $7 \mathrm{~atm}$, non loin de la liquéfaction), aux deux fréquences de 24 et de $36 \mathrm{GHz}$ et à la tem- pérature ambiante $\left(20^{\circ} \mathrm{C}\right.$ environ): Le dispositif utilisé a été conçu et utilisé conformément aux exigences des mesures envisagées.

2. Dispositif et technique de mesure. - La figure 1 est destinée à faciliter la brève description qui suit.

La source est un klystron (VA.98.M pour la bande de $24 \mathrm{GHz}$, VA.97 pour celle de $36 \mathrm{GHz}$ ) modulé par une tension en dents de scie que l'on superpose à la tension continue du réflecteur et dont la fréquence est de $20 \mathrm{~Hz}$. Après avoir traversé un isolateur, l'énẹrgie microonde se dirige, à l'aide d'un coupleur directif, d'une part vers la cellule de mesure (résonateur interférométrique Fabry-Pérot, de facteur de qualité $Q \approx 750000$ ), d'autre part vers une cavité résonnante de référence $C$ (cavité cylindrique conventionnelle, surdimensionnée, $Q \approx 35000$ ).

Un circuit impulsionnel produit, à partir du signal de réponse de la cavité $C$, une brève impulsion $I$ (cf. Fig. 1). Cette dernière sert pour déclencher le balayage horizontal d'un analyseur de signal (HewlettPackard 5480.B). L'analyseur est ici utilisé pour réaliser la moyenne sur un nombre préfixé de mesures 


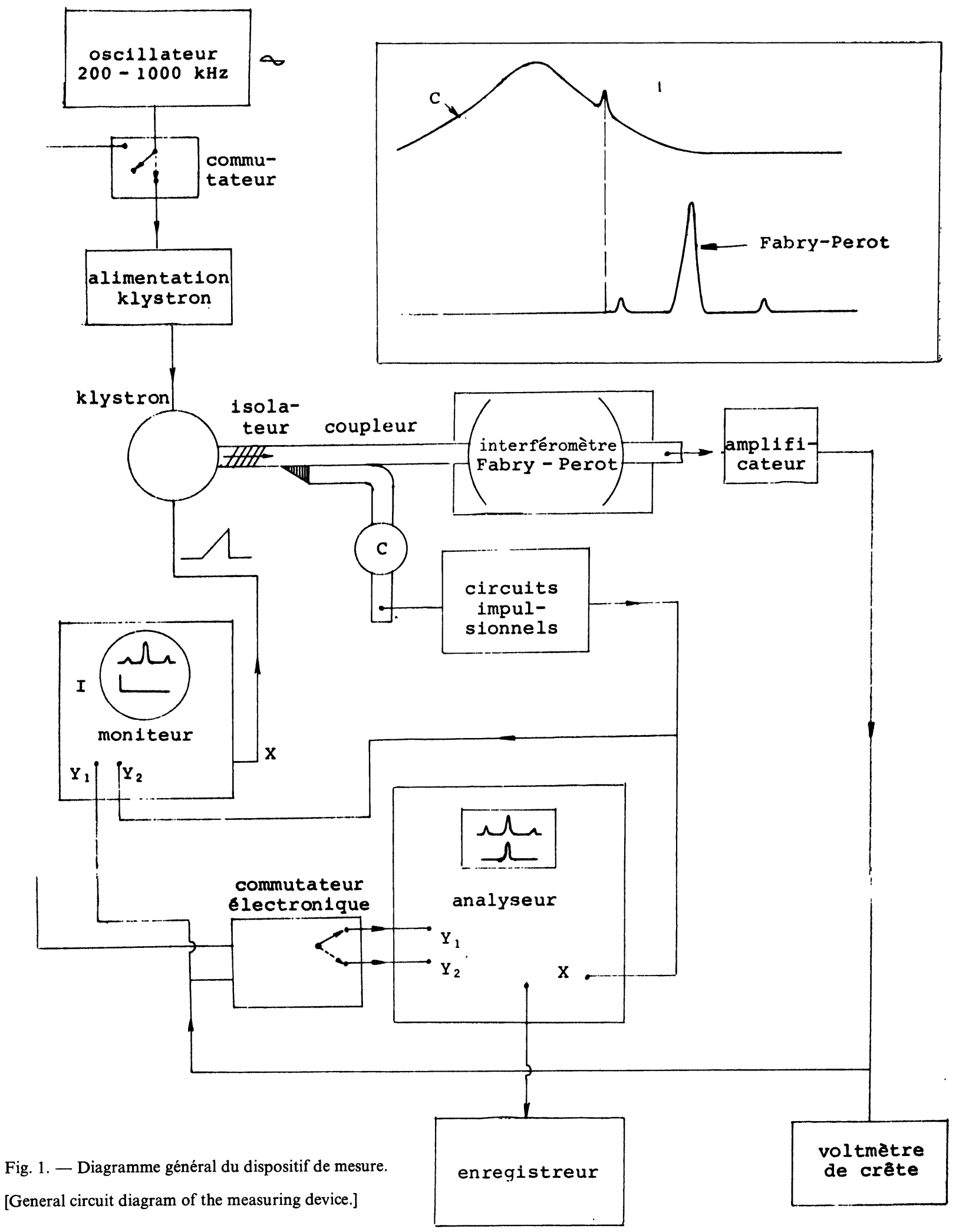

(10 à 30) concernant la largeur de la réponse du résonateur Fabry-Pérot, afin d'améliorer le rapport signal/bruit. Comme il apparaîtra mieux par la suite, la cavité $C$ fournit une référence de fréquence de sorte que le montage ne soit pas affecté des fluctuations du klystron (l'ensemble peut, en fait, être considéré comme une modification du spectromètre différentiel décrit dans [4]).

L'interféromètre Fabry-Pérot est une variante des résonateurs que nous avons réalisés au cours d'un travail antérieur [5]. Il est du type sphérique confocal et se trouve placé dans un cylindre d'acier où l'on 
peut faire le vide et manipuler le gaz étudié, tout en gardant la possibilité de déplacer l'un des miroirs par rapport à l'autre pour accorder le dispositif. Les dimensions du cylindre sont $1 \times 0,40$ mètre environ (Fig. 2).

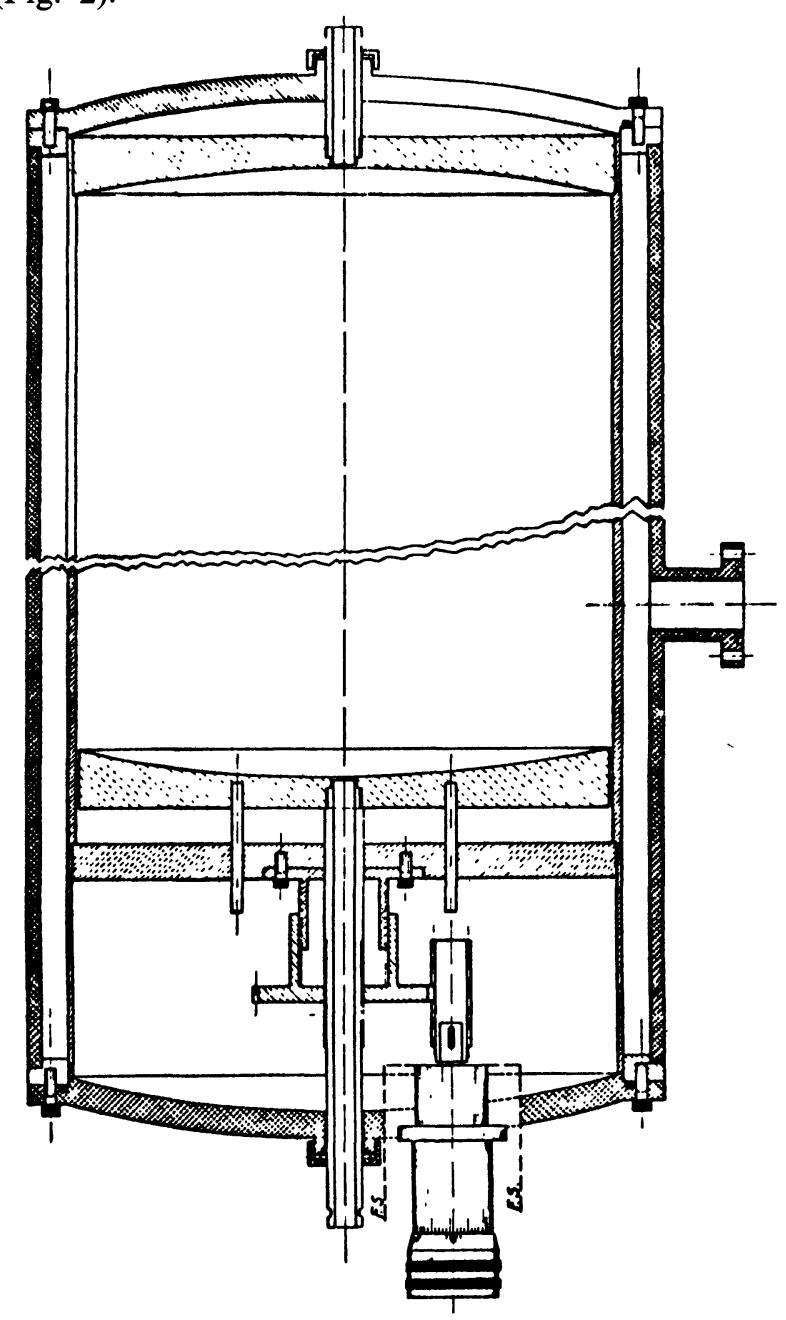

Fig. 2. - Résonateur interférométrique Fabry-Pérot, utilisé comme cellule de mesure.

[Fabry-Pérot interferometer resonator used as measuring cell.]

La fréquence de résonance de l'interféromètre est voisine de celle de la cavité $C$. Au cours d'une période de la modulation en dents de scie, la fréquence du klystron passe successivement par la résonance de la cavité $C$, par la fréquence où l'impulsion $I$ est déclenchée et, ensuite, par la résonance de l'interféromètre. La réponse amplifiée de celui-ci commande la déviation verticale $Y$ de l'analyseur de signal.

Outre la tension en dents de scie, le réflecteur du klystron reçoit par intermittence, moyennant un commutateur électronique, une tension sinusoïdale de plusieurs centaines de kilohertz (300 à $500 \mathrm{kHz}$ pour les mesures ici rapportées). Ainsi, le klystron engendre des bandes latérales qui apparaissent sous forme de pics secondaires sur la réponse de l'interféromètre (par contre, la tension sinusoïdale n'a pratiquement pas d'effet à la sortie de la cavité $C$ à cause de son $Q$ relativement très faible). Les pics latéraux servent à la mesure du facteur de qualité de l'interféromètre. Dans ce but, les signaux de réponse de l'interféromètre (sans ou avec les pics latéraux) sont mémorisés dans l'analyseur de signal dans deux canaux différents et, à la fin de chaque opération de sommation réalisant la moyenne, ils sont enregistrés. On mesure, par ailleurs, la tension maximale amplifiée $V_{0}$ à la sortie de l'interféromètre par le moyen d'un voltmètre à crête.

On sait que les pertes diélectriques $\varepsilon^{\prime \prime}$ (partie imaginaire de la permittivité $\varepsilon=\varepsilon^{\prime}-j \varepsilon^{\prime \prime}$ ) sont liées au facteur $\operatorname{tg} \delta$ par la relation $\operatorname{tg} \delta=\varepsilon^{\prime \prime} / \varepsilon^{\prime}$. Le facteur $\operatorname{tg} \delta$ est déterminé à l'aide des relations suivantes, [6] :

$$
\begin{aligned}
& \operatorname{tg} \delta=\frac{1}{Q_{0}}\left(\left(\frac{P_{0}}{P}\right)^{1 / 2}-1\right) \\
& \operatorname{tg} \delta=\frac{1}{Q}-\frac{1}{Q_{0}}
\end{aligned}
$$

$Q_{0}, P_{0}$ : facteur de qualité et puissance maximale transmise par l'interféromètre vide,

$Q, P:$ les mêmes quantités que l'on mesure en présence du gaz absorbant dans l'enceinte de l'interféromètre.

Nous rappelons que $Q$ est mesuré sur les enregistrements par la technique des bandes latérales, en appliquant la relation $Q=v_{0} / \delta v, v_{0}$ : fréquence de résonance et $\delta v$ : largeur de la réponse de l'interféromètre. En ce qui concerne la puissance $P$, elle est proportionnelle à la tension détectée $V_{0}$ à la sortie de l'interféromètre, pourvu que la détection soit quadratique. Le fait que la détection reste quadratique est vérifié par l'application simultanée des deux relations (1) et (2).

Le bon fonctionnement de l'appareil en vue de mesures de faibles absorptions a été contrôlé par divers tests préliminaires. On a par exemple vérifié, en introduisant dans le résonateur Fabry-Pérot des gaz non absorbants (argon, hélium, etc.), que $Q_{0}$ et $P_{0}$ restent constants quand on fait déplacer le miroir mobile afin de maintenir l'accord de l'interféromètre (dans les limites des variations nécessaires pour mesurer la faible absorption par le propane). On s'assure en même temps que le processus de la sommation n'introduit pas un élargissement appréciable dans le signal de réponse de l'interféromètre.

La sensibilité est donnée par l'absorption minimale effectivement mesurable :

$$
(\operatorname{tg} \delta)_{\min }=\frac{\Delta Q}{Q_{0}^{2}}=\frac{5}{100} \cdot \frac{1}{0,75 \times 10^{6}} \lesssim 10^{-7}
$$

puisque la variation minimale mesurable de $\Delta Q / Q_{0}$ est de $5 \%$ environ. Cette sensibilité, obtenue grâce à la valeur très élevée du facteur $Q_{0}$ de l'interféromètre, est suffisamment grande pour les mesures envisagées. Le grand volume du résonateur FabryPérot rend en même temps les mesures plus stables. 
3. Résultats expérimentaux. - Les pertes diélectriques $\left(\varepsilon^{\prime \prime}\right)$ ont été mesurées à deux fréquences assez éloignées l'une de l'autre (24 et $36 \mathrm{GHz}$ ) en fonction de la pression, jusqu'à $p=7 \mathrm{~atm}$. Comme à la température ambiante le propane se liquéfie aux environs de $9 \mathrm{~atm}$, on doit considérer les corrections des gaz réels par rapport au cas des gaz parfaits.

En ce qui concerne la pression, on doit introduire, dans les relations concernant les gaz parfaits, la pression corrigée $p^{\prime}$ qui, suivant les données pour le propane (cf. Hirschfelder et al., [7]), s'exprime à la température ambiante par :

$$
p^{\prime}=\frac{p}{1-1,7 \times 10^{-2} p} .
$$

Nous admettrons par ailleurs que $\varepsilon^{\prime} \approx 1$ avec une erreur maximale de l'ordre de $3 \%$ vers les plus grandes pressions utilisées. Cette erreur reste pratiquement en marge de l'erreur expérimentale globale.

Les résultats des mesures sont indiqués sur la figure 3. Avant d'aborder la discussion, faisons les trois constatations suivantes :

1) les valeurs mesurées de $\varepsilon^{\prime \prime}$ sont presque doubles de celles rapportées par Maryott et Birnbaum [1], mais on peut encore dire que l'ordre de grandeur reste le même;

2) le comportement de la fonction $\varepsilon^{\prime \prime}\left(p^{\prime}\right)$ est décrit, dans les limites de l'erreur expérimentale, par l'exponentielle :

$$
\varepsilon^{\prime \prime} \times 10^{6}=0,528 \cdot\left(p^{\prime}\right)^{1,33}
$$

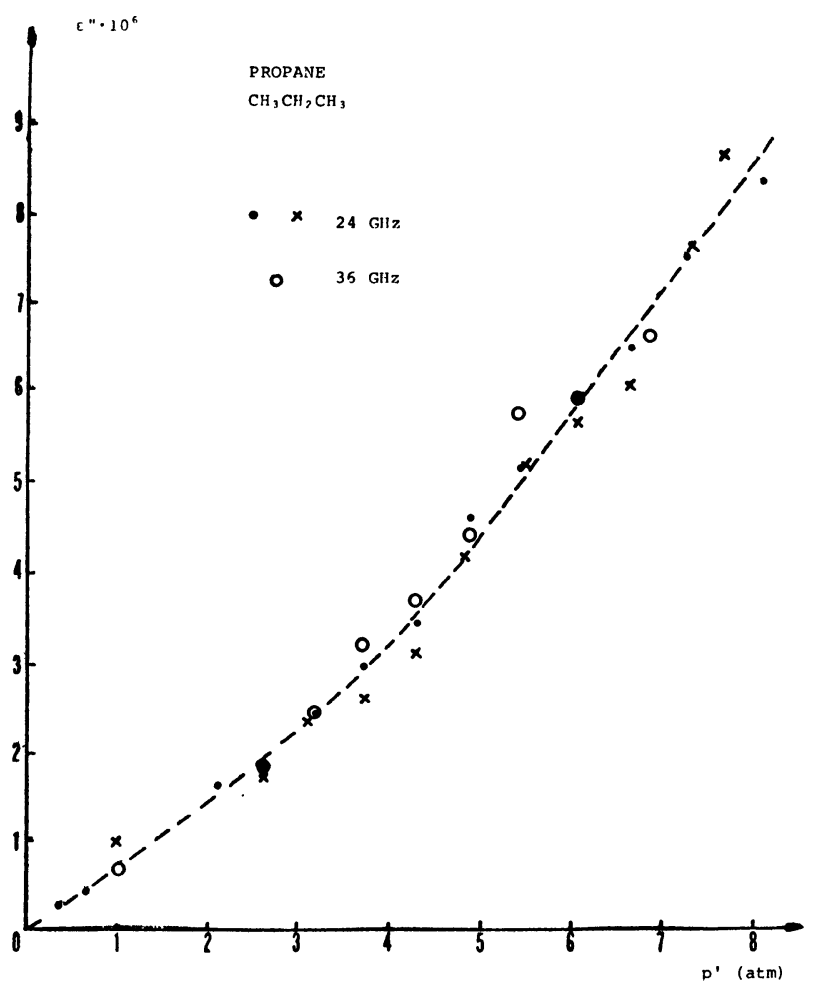

Fig. 3. - Pertes diélectriques $\left(\varepsilon^{\prime \prime}\right)$ en fonction de la pression corrigée $\left(p^{\prime}\right)$ aux fréquences 24 et $36 \mathrm{GHz}$.

[Dielectric losses $\left(\varepsilon^{\prime \prime}\right)$ as a function of the corrected pressure $\left(p^{\prime}\right)$ at the frequencies of 24 and $36 \mathrm{GHz}$.] donc $\varepsilon^{\prime \prime}$ suit une loi entre la loi linéaire et la loi parabolique (et non une loi parabolique comme les auteurs cités tendent à conclure d'après leurs premiers résultats) ;

3) les pertes diélectriques sont pratiquement les mêmes pour les deux fréquences utilisées, c'est-à-dire nous ne retrouvons pas l'accroissement proportionnel à la fréquence indiqué par les mêmes auteurs.

4. Discussion. - On sait (cf. par exemple [8]) que les molécules symétriques à faible moment dipolaire (bien inférieur à un debye) présentent généralement l'absorption du type Debye à un seul temps de relaxation (par inversion de la molécule à fréquence quasi nulle). Tel est par exemple le cas de l'isobutane, pour lequel $\mu=0,132 \mathrm{D}$, [1].

Dans le cas du propane, le moment dipolaire est encore plus faible :

$\mu=0,083 \mathrm{D}$ suivant [2] , 0,084 à $0,085 \mathrm{D}$ suivant [3] ,

mais la molécule est légèrement asymétrique, puisque les trois constantes rotationnelles sont toutes différentes l'une de l'autre [2] :

$$
\begin{gathered}
A=29,207 \mathrm{GHz}, \quad B=8,446 \mathrm{GHz}, \\
C=7,459 \mathrm{GHz}
\end{gathered}
$$

de sorte que le paramètre d'asymétrie ait la valeur :

$$
(2 B-A-C) /(A-C)=-0,91 \text {, }
$$

valeur différente de -1 qui caractérise une molécule symétrique allongée.

Aussi, les mécanismes d'absorption que l'on pourrait envisager pour le propane seraient les suivants (généralement, on pourrait considérer d'autres possibilités : la rotation interne de la molécule par exemple) :

1) absorption résonnante par transitions entre niveaux rotationnels; les fréquences de mesure (24 et $36 \mathrm{GHz}$ ) se trouveraient entre les premières fréquences rotationnelles ou en deça ;

2) absorption non résonnante, attribuée à des transitions rotationnelles à fréquences quasi nulles entre niveaux presque dégénérés (mécanisme décrit par Wilson [9]) ;

3) absorption suivant d'autres mécanismes tels que la création des dipôles temporaires (comme dans le cas du $\mathrm{CO}_{2}$ ) ou encore la répercussion du spectre vibrationnel infrarouge élargi; ces dernières éventualités seront écartées en accord avec les raisonnements formulés dans [1].

Voyons s'il est possible de considérer la totalité de l'absorption mesurée comme absorption non résonnante, du type Debye, à un seul temps de relaxation. 
On aurait dans ce cas [8] :

$$
\begin{aligned}
\operatorname{tg} \delta & =2 s p \frac{v(\Delta v)}{v^{2}+(\Delta v)^{2}}, \quad\left(\operatorname{tg} \delta \simeq \varepsilon^{\prime \prime} \text { pour } \varepsilon^{\prime} \simeq 1\right) \\
s & =\frac{111}{\varepsilon^{\prime} T^{2}}\left\langle\mu_{i j}^{2}\right\rangle=\left(\frac{\operatorname{tg} \delta}{p}\right)_{\max },
\end{aligned}
$$

$\Delta v=g p:$ paramètre d'élargissement $(g$ supposé constant).

Pour qu'il en soit ainsi, le graphique $\left(p^{2} / \varepsilon^{\prime \prime}, p^{2}\right)$ devrait être une droite. La figure 4 de la page suivante (où $p^{\prime}$ est la pression corrigée) montre que ce n'est pas le cas. On peut illustrer quantitativement cette constatation négative en admettant, pour un instant, que l'absorption est représentée par la droite tracée sur la figure 4 . On aurait alors $[10,8]$ :

$$
s=0,985 \times 10^{-6} \mathrm{~atm}^{-1}, \quad g=0,129 \mathrm{GHz} / \mathrm{atm},
$$

et, si l'on appliquait la méthode de calcul donnée dans [10] pour les molécules symétriques, on trouverait qu'à la valeur ci-dessus de $s$ correspond

$$
\mu=0,066 \mathrm{D} \text {. }
$$

Or, le paramètre $\Delta v$ ne devrait pas dépendre de la fréquence (par l'intermédiaire de $g$ ) et, par ailleurs, on sait que $\mu=0,083 \mathrm{D}$.

Par conséquent, seule une partie de l'absorption mesurée peut être du type Debye, l'autre partie, probablement la plus importante (vers les pressions relativement faibles notamment) provenant des mécanismes résonnants. Le comportement de l'absorption totale $\left(\varepsilon^{\prime \prime} \sim p^{1,33}\right.$ et $\varepsilon^{\prime \prime}$ le même pour les deux fréquences utilisées) est compatible avec une telle description. Un calcul quantitatif plus complet nécessiterait des données spectroscopiques détaillées qui ne sont pas disponibles.

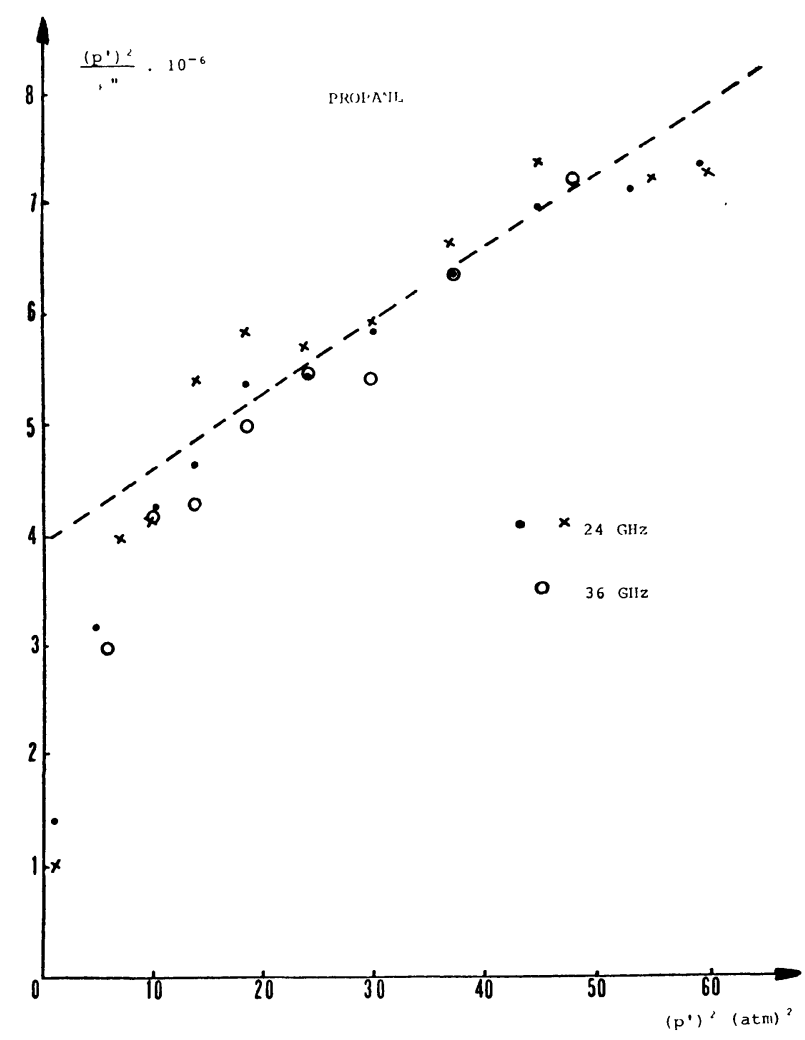

Fig. 4. - Variation de la quantité $\left(p^{\prime}\right)^{2} / \varepsilon^{\prime \prime}$ en fonction de $\left(p^{\prime}\right)^{2}$.

[Variation of the quantity $\left(p^{\prime}\right)^{2} / \varepsilon^{\prime \prime}$ as a function of $\left(p^{\prime}\right)^{2}$.]

La conclusion ci-dessus rejoint celle de Maryott et Birnbaum [1] tout au moins en ce qui concerne la présence d'une absorption résonnante, et ceci malgré les divergences signalées entre les résultats de ces auteurs sur le propane et ceux que nous rapportons dans le présent travail.

\section{Bibliographie}

[1] Maryott, A. A., Birnbaum, G., J. Chem. Phys. 24 (1956) 1022.

[2] Lide, D. A., J. Chem. Phys. 33 (1960) 1514.

[3] Muenter, J. S., Laurie, V. W., J. Chem. Phys. 45 (1966) 855.

[4] Gozzini, A., Battaglia, A., Boudouris, G., Bruin, F., Ilias, D., Onde Elect., suppl. spécial 2 (1958) 430.

Gabalda, F., Chehikian, A., Boudouris, G., Battaglia, A., Ann. Télécommun. 28 (1973) 33.
[5] Battaglia, A., Gozzini, A., Boudouris, G., Nuovo Cimento 69B (1970) 121.

[6] Boudouris, G., Cavités électromagnétiques (Dunod Ed., Paris) 1971.

[7] Hirschfelder, J. O., Curtis, C. F., BiRd, R. B., Molecular theory of gases and liquids (J. Wiley Ed., New York) 1954.

[8] Boudouris, G., Rivista Nuovo Cimento 1 (1969) 1.

[9] Wilson, E. B., J. Phys. Chem. 63 (1959) 1339.

[10] Birnbaum, G., J. Chem. Phys. 27 (1957) 360; 28 (1958) 992. 